\title{
Provider adherence to first antenatal care guidelines and risk of pregnancy complications in public sector facilities: a Ghanaian cohort study
}

Mary Amoakoh-Coleman ${ }^{1,2,3^{*}}$, Kerstin Klipstein-Grobusch ${ }^{2,4}$, Irene Akua Agyepong ${ }^{5,6}$, Gbenga A. Kayode ${ }^{2}$, Diederick E. Grobbee ${ }^{2}$ and Evelyn K. Ansah ${ }^{6}$

\begin{abstract}
Background: Guideline utilization aims at improvement in quality of care and better health outcomes. The objective of the current study was to determine the effect of provider complete adherence to the first antenatal care guidelines on the risk of maternal and neonatal complications in a low resource setting.

Methods: Women delivering in 11 health facilities in the Greater Accra region of Ghana were recruited into a cohort study. Their first antenatal visit records were reviewed to assess providers' adherence to the guidelines, using a thirteen-point checklist. Information on their socio-demographic characteristics and previous pregnancy history was collected. Participants were followed up for 6 weeks post-partum to complete data collection on outcomes. The incidence of maternal and neonatal complications was estimated. The effects of complete adherence on risk of maternal and neonatal complications were estimated and expressed as relative risks (RRs) with their 95\% confidence intervals $(\mathrm{Cl})$ adjusted for a potential clustering effect of health facilities.

Results: Overall, 926 women were followed up to 6 weeks post-partum. Mean age (SD) of participants was 28.2 (5.4) years. Complete adherence to guidelines pertained to the care of $48.5 \%$ of women. Incidence of preterm deliveries, low birth weight, stillbirths and neonatal mortality were $5.3,6.1,0.4$ and $1.4 \%$ respectively. Complete adherence to the guidelines decreased risk of any neonatal complication [0.72 $(0.65-0.93) ; p=0.01]$ and delivery complication [0.66 (0.44-0.99), $p=0.04]$.

Conclusion: Complete provider adherence to antenatal care guidelines at first antenatal visit influences delivery and neonatal outcomes. While there is the need to explore and understand explanatory mechanisms for these observations, programs that promote complete adherence to guidelines will improve the pregnancy outcomes.
\end{abstract}

Keywords: Adherence, Antenatal care, First antenatal visit, Maternal, Neonatal, Outcomes

\section{Background}

With respect to Millennium Development Goal 5 (MDG 5), substantial progress has been achieved in almost all regions, except in sub-Saharan Africa, which could not reach the set target of reducing maternal mortality by $75 \%$ by 2015 [1]. Given Ghana's progress

\footnotetext{
*Correspondence: menba19@yahoo.com

'Postdoctoral Unit, Noguchi Memorial Institute for Medical Research, University of Ghana, Legon, Accra, Ghana

2Julius Global Health, Julius Center for Health Sciences and Primary Care,

University Medical Centre Utrecht, Utrecht, The Netherlands

Full list of author information is available at the end of the article
}

so far, maternal mortality was estimated to be reduced to only 340 per 100,000 by the end of 2015 instead of the MDG target of 185 per 100,000 [2-4].

Efforts made to help Ghana meet the MDG included adoption of the Safe Motherhood Initiative (SMI) launched globally in 1987, together with other policy introductions such as free antenatal care for all pregnant women in 1998 and exempting all users from delivery fees in health facilities in 2003 [5].

One of the public health interventions aimed at alleviating complications of pregnancy and childbirth is antenatal 
care $[6,7]$. The first antenatal care visit offers the opportunity to identify women who are likely to have unfavourable pregnancy outcomes [8]. Guidelines for antenatal care exist to support the health care provider to maximize this potential.

Adherence to clinical guidelines is important in ensuring uniformity of clinical care, as well as maintaining quality service provision to patients based on their specific needs [9]. This has also been shown to lead to improved clinical outcomes [10-12]. Studies measuring the effects of guidelines on quality of care have shown significant improvements in the process of care [13-16]. Other studies focusing on effects on patient health outcomes [17-21] have demonstrated improved outcomes with increased adherence [17, 18, 22-24]. Most of these studies however have been conducted in advanced resource settings, and focused on non-obstetric medical and surgical conditions. In Ghana, studies that have evaluated adherence to guidelines are limited [25-27] with few focusing on antenatal care guidelines.

Adherence to guidelines has been shown to vary considerably in many settings and often remain low [8, 26-28], translating into poor health outcomes. As part of the SMI implementation in Ghana, various tools including protocols and guidelines such as the National Safe Motherhood Service Protocol (SMP) were developed to assist health workers in caring for pregnant women.

Our objective was to determine the effect of provider complete adherence to the first antenatal care guidelines on the risk of maternal and neonatal complications.

\section{Methods}

\section{Study design}

A cohort study with both retrospective and prospective arms was conducted.

\section{Study setting}

The study was conducted in the Greater Accra Region (GAR) of Ghana. The region is about $90 \%$ urban, and is served by both public and private facilities. About $97 \%$ of pregnant women here attend antenatal clinic at least once during their pregnancy and skilled attendance at delivery is around $84 \%$. About $62 \%$ of all health facility deliveries in the region take place in the public sector. The public sector comprises of one teaching hospital, a regional hospital and nine district and sub-metropolitan hospitals. There are ten polyclinics, 31 health centres, some community clinics and three Community Health Planning and Services (CHPS) compounds. The polyclinics are primary health care facilities like the health centres, usually sited in urban and populous areas and provide both general and specialist services. They also have more than one doctor unlike the health center which usually has one doctor or in some cases manned by a medical assistant. CHPS compounds operate at the community level, with midwives or community health nurses, either through home visits or clients going to the compounds. There are several private hospitals operating in the region. All these health facilities offer antenatal and delivery services, though the smaller facilities refer complications to the bigger health facilities. The National Health Insurance Scheme (NHIS) is operational in all the public as well as in most of the private facilities.

\section{Selection of districts and facilities}

We randomly selected participants from the different levels of public health care (variable "type of facility") across the Greater Accra region. All districts and submetropolises in the region were grouped into those with a district or sub-metropolitan hospital (eight in number) and those without hospitals (12 in total) and then five districts were randomly selected by balloting with replacement from each group. By this process, the names of the districts in each group were written on a piece of paper, folded and put in a box. After shuffling by an independent person, five districts were picked one at a time, replacing the picked district in the box each time before picking the next to ensure equal chance of selection for all. The district hospitals in the selected districts with hospitals were included in our study. In those districts without hospitals all the primary level care facilities offering both antenatal and delivery services were included for random sampling and one was selected from each district." We also included the regional hospital which is in a sub-metropolis different from the selected districts/metropolises. Thus in all, 11 health facilities (one regional and five district/sub-metropolitan hospitals, four polyclinics and a health center) were randomly selected from 11 districts/sub-metropolises for the study.

\section{Sample size and recruitment of women}

The sample size for the cohort study was based on a prevalence of pregnancy complications of 6\% [29] using Open Epi calculator for estimation. In the absence of any documented evidence in the setting, we assumed that the complication rate will be twice as high amongst the unexposed group (incomplete adherence). To detect a twosided significance difference at $95 \%$ confidence interval at a power of $80 \%$, and a one-to-one ratio of exposure to non-exposure, a sample of 372 women was required per exposure group. The minimum total sample size therefore required for both arms was 744 women.

Participants were recruited at delivery and once they met the inclusion criteria, informed consent was obtained. The inclusion criteria included the following: participant 18 years or older; participant had at least one antenatal 
clinic visit in a health facility during the current pregnancy; participant had first antenatal clinic visit at gestational age less than or equal to 5 months and participant had first antenatal clinic visit at the facility of delivery or in one of the sampled facilities for this study.

From each of the eleven (11) facilities sampled for the study, we recruited a minimum of 68 women, a minimum of 34 women exposed to complete provider adherence and a minimum of 34 unexposed women. We continued recruitment irrespective of adherence status until we had at least 34 women in either group.

\section{Data collection processes and tools}

Every woman delivering at the facility on any day and who met the inclusion criteria, and provided informed consent for participation, was enrolled into the study.

At recruitment, a record review of their first antenatal clinic visit from the maternal health record book was retrospectively carried out using a checklist. Data on socio-demographic characteristics, potential confounders such as timing of first antenatal visit, number of antenatal clinic attendance and previous pregnancy history, as well as the 13 variables on guideline requirements were collected. Records of subsequent antenatal clinic visits were also reviewed for any complications developed and identified during the pregnancy. Finally, data on delivery outcomes was collected from both the maternal health record book and delivery register and notes. Participants' telephone numbers were linked to their study identification numbers (IDs) for follow up.

In the prospective arm, all women and their neonates were followed up till 6 weeks postpartum to complete data collection on outcomes. Follow up was at the health facility during post-natal care visit at 6 weeks postpartum and also by phone. Participants received a phone call 3 weeks post-partum, and at 6 weeks, they were followed up at the postnatal clinic. If they were not available there, they received another phone call. Those who could not be reached by any of these means were treated as lost to follow up. A facility audit was conducted to assess facility factors such as the availability of personnel, services, infrastructure, logistics and supplies that are needed to support adherence to the guidelines at facility level.

The assumption was made that any information on history, examination, laboratory examination and treatment available is what was recorded in the maternal health record book. Information on any service not recorded, was deemed not to have been delivered [30].

\section{Variables}

The outcome variables studied were all maternal and neonatal complications during antenatal, delivery and post-partum periods. Maternal complications were i. any antenatal complication (defined as having at least one of the following: anemia in pregnancy, pregnancy induced hypertension/pre-eclampsia/eclampsia, malaria in pregnancy and antepartum vaginal bleeding), ii. Any antenatal complication excluding anemia in pregnancy,, iii. Caesarean section, iv. Delivery complication (defined as having at least one of the following: pregnancy induced hypertension/pre-eclampsia/eclampsia, ruptured uterus, obstructed labour, vaginal tears and perineal tears), v. post-delivery complication (defined as having at least one of the following: postpartum haemorrhage, anemia, malaria and sepsis), vi. Maternal mortality, vii. Any maternal complication (which is defined as having at least one of the listed maternal complications). Neonatal complications were preterm, post-maturity, low birth weight, neonatal jaundice, asphyxia, still birth and neonatal mortality.

The determinants were type of facility, client's sociodemographics (age, educational level, marital status and employment status), and client's prenatal factors (parity, trimester at first antenatal care visit, previous pregnancy history and number of times antenatal clinic was attended during pregnancy). Provider adherence levels were defined as complete or incomplete.

\section{Measuring adherence}

Several studies have measured adherence using a scoring system, based on available guidelines or protocol requirements [21, 31-33]. In this study, a thirteen-point checklist was used to score provider adherence to first antenatal visit guidelines. Adherence to first antenatal guidelines was used as a proxy for adherence to guidelines in general. The questions on the checklist were based on the requirements for first antenatal visit as per the SMP for Ghana, which is also consistent with the national treatment guidelines for first antenatal visit. They related to if: Age recorded; Parity recorded; Gestational age at booking recorded; Last pregnancy history if applicable recorded; Medical, surgical or family history recorded; Weight recorded; Blood pressure recorded; Abdomen examined; Haemoglobin test done; Urine test done; Iron supplement given; Tetanus injection given or status recorded; Intermittent preventive treatment of malaria (IPTp) given. Eleven of the variables on the checklist were referred to as "mandatory variables" while two of them were referred to as "optional" variables. The "optional variables may not be due at the first antenatal visit, depending on the woman's gestational age and therefore do not influence the adherence categorization. These are the "last pregnancy history if applicable" and "Intermittent Presumptive Treatment in pregnancy (IPTp) given if woman is due". IPTp is indicated for women in the second and third trimesters only. We included the "optional" variables in order to describe 
how they contribute to antenatal care quality. Every record reviewed was assessed to see how many of the 13 variables were actually adhered to by the provider at the first antenatal visit.

Each variable adhered to, scored a point of 1 while non-adherence scored 0. A total score of 11-13 (including a score of 1 to all the 11 mandatory variables) was classified as complete adherence to guidelines. Nonadherence to any of the mandatory variables was classified as incomplete adherence. The adherence checklist, scoring criteria and entire methodology for the study has been published elsewhere [34] and is provided as Additional file 1.

\section{Data analysis}

Descriptive analysis of participants' socio-demographic information and previous pregnancy history was conducted by use of frequencies and chi-square analysis. Adherence to guidelines was computed by calculating the proportion of women whose first antenatal clinic visits met the criteria for complete adherence. Possible associations of adherence status with some patient characteristics were assessed using chi-square. Incidences of maternal and neonatal complications were estimated in percentages. Determinants of maternal and neonatal complications, as well as the effect of complete adherence on pregnancy outcomes were estimated and expressed as relative risks (RRs) with their 95\% confidence intervals $(\mathrm{CI})$. To correct for potential clustering effect due to our sampling strategy, we used generalized linear equation estimation in calculating RRs. We also adjusted for variables such as trimester of first ANC visit, maternal age, parity, previous pregnancy complication and any antenatal complication where appropriate. Significance was determined at $p$-value $<0.05$. Data analysis was carried out using IBM SPSS Statistics for Windows, Version 20.0. Armonk, NY: IBM Corp.

\section{Results}

A total of 946 women were recruited and 926 of them were followed up to 6 weeks post-partum from December 2013 to May 2014. We recruited more women than the minimum required because the minimum number of women per each group had to be satisfied. In places where more women with complete adherence were included, we continued recruitment until we reached the minimum of 34 for the incomplete adherence group, and vice versa. Figure 1 is a flow chart of participants' recruitment and follow-up in the study.

Fifty-six percent of the women were seen at six hospitals, while $36.0 \%$ were seen at four polyclinics and $8.0 \%$ at a health center.

\section{Adherence to first antenatal care guidelines}

Overall, complete adherence to guidelines pertained to the care of only $48.5 \%$ [95\% CI (45.3-51.7\%)] of all participants in our sample, during their first antenatal clinic visit. The range was 33.8 to $61.7 \%$ amongst the

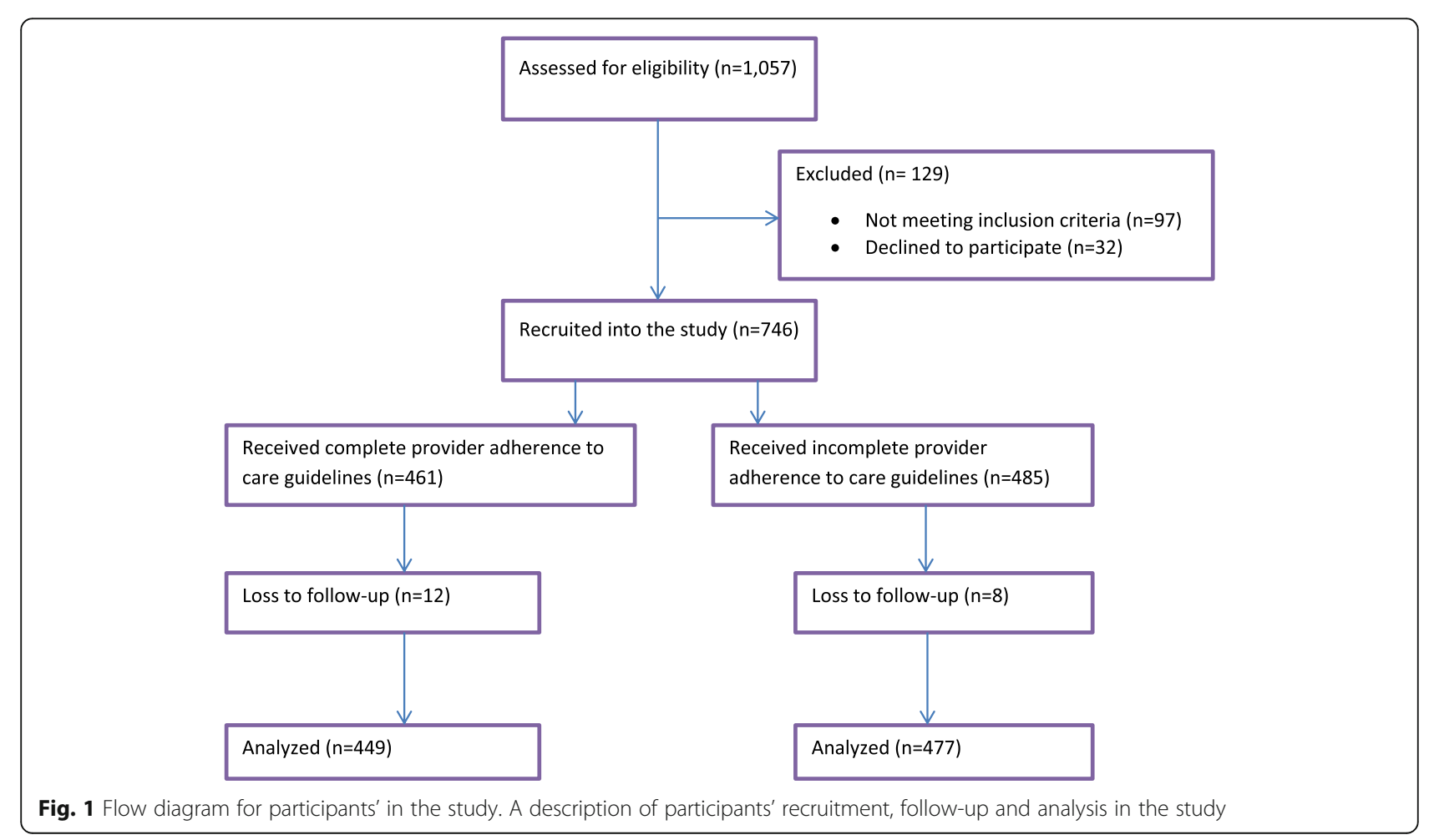


facilities sampled. Complete adherence to guidelines was higher amongst women seen at the polyclinics [52.9\% $(47.5-58.2 \%)]$ than those seen in hospitals [47.8\% $(43.5-52.1 \%)]$ and the health center [34.2\% (22.8-44.8\%)].

\section{Participants' baseline characteristics}

The mean age (SD) of participants was 28.2 (5.4) years. About $85 \%$ of them were aged $20-35$ years. Less than half of participants had secondary education, with 11.8 and $19.3 \%$ having no education and primary education respectively. Married women made up $72.6 \%$ of all participants and most of the women $(84.9 \%)$ were employed, $68.1 \%$ had their first antenatal visit during the second trimester and $78.1 \%$ attended at antenatal clinic at least four times during the pregnancy. Only $7.7 \%$ of the women had a history of previous pregnancy complication and $45.6 \%$ of the women were anemic at their first antenatal visit. Details of the baseline characteristics for the two groups of participants are given in Table 1.

\section{Risk of maternal and neonatal complications}

Table 2 shows the incidence of maternal and neonatal complications. Overall, $68.1 \%$ of the women developed antenatal complications. As many as $59.4 \%$ of the women had anemia during the pregnancy and 15.5\% developed anemia after the first antenatal visit. Incidence of pregnancy-induced hypertension and its complications and post-partum hemorrhage were 5.3 and 2.1\% respectively. Risk for any pregnancy, delivery or postpartum complication was $68.6 \%$. There was no maternal mortality amongst the study participants. Incidence of preterm deliveries, low birth weight babies, stillbirths and neonatal mortality were 5.3, 6.1, 0.4 and $1.4 \%$ respectively. There were differences in risks of most of the complications amongst the two adherence groups, but these were not statistically significant.

In the univariable analysis, provider adherence significantly influenced both neonatal and any maternal complication (antenatal, delivery or post-partum) (Table 3). Neonatal complications were reduced by almost $30.0 \%$ amongst women whose first ANC care was standard as per the guidelines while delivery related complication for such women was reduced by about $40.0 \%$. Controlling for potential confounders in a multivariable analysis only slightly altered risk estimates for neonatal [0.72 (0.56$0.93), p=0.01]$, and delivery related complications [0.66 (0.33-0.99), $p=0.04$ ], (Table 4).

\section{Discussion}

\section{Main findings}

Key findings of this study are that provider adherence to first antenatal care guidelines is low (48.5\%), most women register for first antenatal care in the second trimester and
Table 1 Baseline characteristics of study participants and association of these characteristics with levels of provider adherence to first antenatal care (ANC) guidelines

\begin{tabular}{|c|c|c|c|c|}
\hline \multirow[t]{2}{*}{ Variable } & $\begin{array}{l}\text { Frequency } \\
(\%)\end{array}$ & $\begin{array}{l}\text { \% Incomplete } \\
\text { adherence }\end{array}$ & $\begin{array}{l}\text { \% Complete } \\
\text { adherence }\end{array}$ & $\begin{array}{l}\text { Cluster } \\
\text { adjusted }\end{array}$ \\
\hline & $N=926$ & $N=477$ & $N=449$ & $p$-value \\
\hline Total sample & & 51.5 & 48.5 & \\
\hline Facility type & & & & $<0.01$ \\
\hline Hospital & $519(56.0)$ & 52.2 & 47.8 & \\
\hline Polyclinic & $333(36.0)$ & 47.1 & 52.9 & \\
\hline Health center & $74(8.0)$ & 65.8 & 34.2 & \\
\hline Age category & & & & 0.01 \\
\hline$<20$ years & $52(5.6)$ & 73.1 & 26.9 & \\
\hline 20-35 years & $790(85.5)$ & 50.5 & 49.5 & \\
\hline$>=35$ years & $82(8.9)$ & 47.6 & 52.4 & \\
\hline Parity & & & & 0.01 \\
\hline 0 & $258(27.9)$ & 76.7 & 22.7 & \\
\hline $1-2$ & $475(51.3)$ & 40.8 & 59.2 & \\
\hline $3-4$ & $162(17.5)$ & 43.2 & 56.8 & \\
\hline$>4$ & $31(3.3)$ & 48.4 & 51.6 & \\
\hline Education & & & & 0.87 \\
\hline None & 109 (11.8) & 47.7 & 52.3 & \\
\hline Primary & 179 (19.3) & 47.5 & 52.5 & \\
\hline Secondary & $423(45.7)$ & 51.8 & 48.2 & \\
\hline Tertiary & 163 (17.7) & 57.1 & 42.9 & \\
\hline Other & $46(5.0)$ & 47.8 & 52.2 & \\
\hline $\begin{array}{l}\text { Trimester at first } \\
\text { ANC }\end{array}$ & & & & 0.05 \\
\hline First & $282(30.5)$ & 57.8 & 42.2 & \\
\hline Second & $631(68.1)$ & 48.0 & 52.0 & \\
\hline $\begin{array}{l}\text { Number of times } \\
\text { ANC attended }\end{array}$ & & & & $<0.01$ \\
\hline 1 & $44(4.8)$ & 79.5 & 20.5 & \\
\hline $2-3$ & $137(14.8)$ & 48.9 & 50.7 & \\
\hline$>=4$ & 723 (78.1) & 50.1 & 49.9 & \\
\hline Marital status & & & & 001 \\
\hline Single & $100(10.8)$ & 64.0 & 20.5 & \\
\hline Married & $672(72.6)$ & 48.5 & 51.5 & \\
\hline Formerly married & $12(1.3)$ & 58.3 & 41.7 & \\
\hline Living together & $122(13.2)$ & 54.9 & 45.1 & \\
\hline Employment & & & & 0.01 \\
\hline No & $141(15.2)$ & 66.0 & 34.0 & \\
\hline Yes & 777 (84.9) & 48.8 & 51.2 & \\
\hline $\begin{array}{l}\text { Previous pregnancy } \\
\text { history }\end{array}$ & & & & 0.04 \\
\hline $\begin{array}{l}\text { No } \\
\text { complication }\end{array}$ & $853(92.1)$ & 51.2 & 48.8 & \\
\hline Mean age (SD) & $28.15(5.4)$ & 27.25 (5.6) & $29.11(5.0)$ & $<0.01$ \\
\hline
\end{tabular}


Table 2 Risk of antenatal, delivery and postpartum complications amongst cohort and comparison of risks between the 2 adherence groups

\begin{tabular}{|c|c|c|c|c|}
\hline Complication & $\begin{array}{l}\text { Incidence (\%) } \\
N=926\end{array}$ & $\begin{array}{l}\text { Complete adherence } \\
N=449\end{array}$ & $\begin{array}{l}\text { Incomplete adherence } \\
N=477\end{array}$ & $\begin{array}{l}\text { Cluster adjusted } \\
p \text {-value }\end{array}$ \\
\hline \multicolumn{5}{|l|}{ Maternal } \\
\hline Malaria in pregnancy & $113(12.2)$ & $56(12.5)$ & $57(11.9)$ & 0.81 \\
\hline Antepartum vaginal bleeding & $10(1.1)$ & $3(0.7)$ & $7(1.5)$ & 0.24 \\
\hline $\mathrm{PIH} /$ pre-eclampsia/eclampsia & $49(5.3)$ & $23(5.1)$ & $26(5.5)$ & 0.82 \\
\hline Anemia in pregnancy & $550(59.4)$ & $269(59.9)$ & $281(58.9)$ & 0.12 \\
\hline Anemia developed after first antenatal visit & $144(15.5)$ & $67(14.9)$ & $77(16.1)$ & 0.36 \\
\hline Any antenatal complication & $631(68.1)$ & $308(68.6)$ & $328(68.7)$ & 0.88 \\
\hline Any antenatal complication (non-anemia) & $207(22.4)$ & $99(22.0)$ & $108(22.6)$ & 0.83 \\
\hline Delivery by Caesarean section & $132(14.3)$ & $65(14.5)$ & $67(14.0)$ & 0.84 \\
\hline Post-partum hemorrhage & $19(2.1)$ & $6(1.3)$ & $13(2.7)$ & 0.14 \\
\hline Delivery/post-delivery complication & $41(4.4)$ & $17(3.8)$ & $24(5.0)$ & 0.82 \\
\hline Any pregnancy related complication & $635(68.6)$ & $310(69.0)$ & $325(68.1)$ & 0.87 \\
\hline Any pregnancy related complication (non-anemia) & $229(24.4)$ & $108(24.1)$ & $121(25.4)$ & 0.56 \\
\hline Maternal mortality & $0(0.0)$ & $0(0.0)$ & $0(0.0)$ & \\
\hline \multicolumn{5}{|l|}{ Neonatal } \\
\hline Preterm & $49(5.3)$ & $23(5.1)$ & $26(5.5)$ & 0.31 \\
\hline Post-maturity & $88(9.5)$ & $36(8.0)$ & $52(10.9)$ & 0.31 \\
\hline Low birth weight & $57(6.1)$ & $19(4.2)$ & $36(7.5)$ & 0.21 \\
\hline Neonatal jaundice & $58(6.3)$ & $29(6.5)$ & $29(6.1)$ & 0.21 \\
\hline Asphyxia/difficulty in breathing & $39(4.2)$ & $15(3.3)$ & $34(7.1)$ & 0.21 \\
\hline Stillbirths & $4(0.4)$ & $2(0.4)$ & $2(0.4)$ & 0.21 \\
\hline Any neonatal complication & $146(15.8)$ & $60(13.4)$ & $86(18.0)$ & 0.05 \\
\hline Neonatal mortality (All cause) & $13(1.4)$ & $6(1.3)$ & $7(1.5)$ & 0.87 \\
\hline
\end{tabular}

complete adherence to first antenatal guidelines reduced the risk of delivery and any neonatal complication.

\section{Strengths and limitations}

Adherence to first antenatal care guidelines was retrospectively scored from participants' records. It is therefore not possible that provider practice changed because of the study, thus reflecting everyday provider practice of adherence to guidelines. Loss of follow-up was minimal at $2.1 \%$. We however recognize some limitations for our study. The fact that we recorded zero maternal deaths in our study is not reflective of the situation in Greater Accra, and might purely be due to the fact most of our participants are those who survived pregnancy and delivery. Also, since only women receiving care in public facilities were included in the study, generalizability of the results to service delivery in private practice is limited.

\section{Interpretation}

Antenatal care as well as skilled attendance at delivery (which all participants in our study had) have been shown to improve maternal and neonatal outcomes [35].
Provider adherence to first antenatal care guidelines was observed to be low in our study in contrast to what other studies [36-38] conducted in advanced settings found. However, one earlier study found limited use of maternal health guidelines in Ghana, Burkina Faso and Tanzania [39]. Previous studies from Ghana identified gaps in quality of care of maternal, new-born and child health, including antenatal care $[40,41]$, which may be explained by low adherence to guidelines and subsequent suboptimal quality of care provided to pregnant women.

The current study demonstrates the significant role of quality of care in translating access to antenatal care to good outcomes. Complete adherence to first antenatal guidelines resulted in reduced risk of delivery and neonatal complications. Guidelines can only lead to improved or quality care if they are translated into daily provider practice [42]. As they continue to provide service, clinicians may not even be aware of their lack of adherence to available guidelines [43]. It has been established that active steps, such as reminders, are necessary to translate clinical practice guidelines into daily practice [9, 42, 44]. The process of engagement of and support for providers of 
Table 3 Univariable association between independent variables and antenatal, delivery and post-partum complications among study participants

\begin{tabular}{|c|c|c|c|c|c|c|c|c|}
\hline Independent factor & $\begin{array}{l}\text { Antenatal } \\
\text { complication } \\
\text { RR }(95 \% \mathrm{Cl})\end{array}$ & $p$-value & $\begin{array}{l}\text { Delivery/ } \\
\text { postpartum } \\
\text { complication } \\
\text { RR }(95 \% \mathrm{Cl})\end{array}$ & $p$-value & $\begin{array}{l}\text { Any maternal } \\
\text { complication } \\
\text { RR }(95 \% \mathrm{Cl})\end{array}$ & $p$-value & $\begin{array}{l}\text { Neonatal } \\
\text { complication } \\
\text { RR }(95 \% \mathrm{Cl})\end{array}$ & $p$-value \\
\hline Complete adherence & $1.02(0.70-.50)$ & 0.90 & $0.73(0.48-1.11)$ & 0.14 & $0.64(0.43-0.94)$ & 0.03 & $0.73(0.55-0.96)$ & 0.03 \\
\hline Employment (Yes) & $0.72(0.4-1.10)$ & 0.13 & $2.17(1.17-4.01)$ & 0.01 & $1.19(0.63-2.95)$ & 0.58 & $0.89(0.60-1.32)$ & 0.56 \\
\hline \multicolumn{9}{|l|}{ Age categories } \\
\hline$<20$ years & $t$ & & $t$ & & $t$ & & $t$ & \\
\hline 20-35years & $1.20(0.76-1.91)$ & 0.44 & $0.60(0.26-1.40)$ & 0.24 & $0.42(0.28-0.64)$ & $<0.01$ & $0.60(0.31-1.34)$ & 0.12 \\
\hline$>35$ years & $0.74(0.39-1.41)$ & 0.36 & $1.72(0.64-4.63)$ & 0.29 & $1.04(0.68-1.58)$ & 0.85 & $0.37(0.12-1.13)$ & 0.08 \\
\hline \multicolumn{9}{|l|}{ Parity } \\
\hline 0 & $t$ & & $t$ & & $t$ & & $t$ & \\
\hline $1-2$ & $0.91(0.71-1.71)$ & 0.48 & $1.56(0.51-4.73)$ & 0.43 & & & $0.68(0.48-0.95)$ & 0.03 \\
\hline $3-4$ & $1.49(0.97-2.31)$ & 0.07 & $2.80(1.47-5.35)$ & $<0.01$ & & & $0.68(0.43-1.07)$ & 0.10 \\
\hline$>4$ & $0.72(0.31-1.71)$ & 0.46 & $1.11(0.38-3.20)$ & 0.85 & & & $0.59(0.25-6.41)$ & 0.24 \\
\hline \multicolumn{9}{|l|}{$\begin{array}{l}\text { Trimester of first ANC } \\
\text { attendance }\end{array}$} \\
\hline First & $t$ & & t & & $t$ & & $t$ & \\
\hline Second & $1.10(0.72-1.68)$ & 0.65 & $0.87(0.35-2.17)$ & 0.77 & $0.82(0.41-1.69)$ & 0.57 & $0.86(0.53-1.38)$ & 0.53 \\
\hline $\begin{array}{l}\text { Number of times } \\
\text { ANC attended }(>=4)\end{array}$ & $0.73(0.28-1.84)$ & 0.50 & $0.46(0.11-1.98)$ & 0.30 & $0.63(0.17-2.34)$ & 0.49 & $1.01(0.19-1.77)$ & 0.33 \\
\hline $\begin{array}{l}\text { Previous delivery } \\
\text { complication (Yes) }\end{array}$ & $1.11(0.64-1.94)$ & 0.71 & $1.13(0.50-2.59)$ & 0.76 & $1.11(0.31-3.91)$ & 0.88 & $1.24(1.81-1.92)$ & 0.32 \\
\hline $\begin{array}{l}\text { Any antenatal } \\
\text { complication }\end{array}$ & NA & & $0.87(0.54-1.37)$ & 0.54 & NA & & $1.55(1.10-2.19)$ & 0.01 \\
\hline $\begin{array}{l}\text { Antenatal } \\
\text { complication } \\
\text { (non-anemia) }\end{array}$ & NA & & $1.92(1.09-3.38)$ & 0.02 & NA & & $2.67(1.86-3.83)$ & $<0.01$ \\
\hline
\end{tabular}

ANC denotes antenatal clinic, $C l$ denotes confidence interval, NA denotes not applicable, $R R$ denotes relative risk

Table 4 Effect of complete adherence on risk of pregnancy complications

\begin{tabular}{|c|c|c|c|c|}
\hline Complication & $\begin{array}{l}\text { Crude RR } \\
95 \% \text { Confidence interval }\end{array}$ & $p$-value & $\begin{array}{l}\text { aAdjusted RR } \\
\text { 95\% Confidence interval }\end{array}$ & $p$-value \\
\hline Neonatal & $0.73(0.55-0.96)$ & 0.03 & $0.72^{c}(0.56-0.93)$ & 0.01 \\
\hline Any maternal & $1.01(0.67-1.48)$ & 0.97 & $1.00^{\mathrm{b}}(0.67-1.48)$ & 0.99 \\
\hline Antenatal & $1.02(0.70-1.50)$ & 0.90 & $1.02^{\mathrm{b}}(0.69-1.52)$ & 0.92 \\
\hline Antenatal (non-anemia) & $0.96(0.75-1.23)$ & 0.73 & $0.90^{\mathrm{b}}(0.66-1.22)$ & 0.45 \\
\hline PIH/pre-eclampsia/eclampsia & $0.92(0.62-1.37)$ & 0.69 & $0.95^{\mathrm{b}}(0.57-1.58)$ & 0.84 \\
\hline Antepartum vaginal bleeding & $0.45(0.17-1.21)$ & 0.11 & $0.48^{\mathrm{b}}(0.16-1.43)$ & 0.19 \\
\hline Malaria in pregnancy & $1.05(0.70-1.58)$ & 0.80 & $1.02^{\mathrm{b}}(0.68-1.53)$ & 0.92 \\
\hline Delivery & $0.64(0.43-0.94)$ & 0.03 & $0.66^{\mathrm{b}}(0.44-0.99)$ & 0.04 \\
\hline Post-partum & $0.71(0.28-1.77)$ & 0.46 & $0.72^{\mathrm{b}}(0.33-1.59)$ & 0.42 \\
\hline
\end{tabular}

$P I H$ denotes pregnancy induced hypertension, $R R$ denotes relative risk

${ }^{\mathrm{a} A l l}$ RR adjusted for possible clustering effect due to sampling strategy

${ }^{\mathrm{b}}$ Estimate adjusted for marital status, employment, number of times ANC was attended, trimester of first antenatal visit, maternal age, parity and previous pregnancy complication

'Estimate adjusted for marital status, employment, number of times ANC was attended trimester of first antenatal visit, maternal age, parity, previous pregnancy complication and any antenatal complication 
maternal and neonatal care with regards to utilization of clinical guidelines can be enhanced through a mechanism of regular monitoring and providing feedback to them as has been done in other contexts [45]. Programs to improve adherence to guidelines that are fashioned around a continuous educational framework for providers are likely to transform provider practice [46] and will subsequently improve the outcome of neonatal service delivery.

Provider adherence to guidelines however did not significantly influence the risk of any maternal complications, although it specifically reduced the risk of delivery related complications. Perhaps other mechanisms better explain maternal outcomes apart from adherence. It must also be recognized that perhaps the frequent antenatal clinic attendance allows providers to make up for what they missed out during the first antenatal visit. Basic, skilled and ultimately safe intrapartum care is noted to be important in addressing maternal and neonatal morbidity and mortality [2, 47], and any intervention of the process that results in better outcomes should be encouraged. Evidence based practice intrapartum is essential as one systematic review has shown [48]. Thus mechanisms to enhance provider adherence to both ANC and delivery guidelines should be instituted at all levels of care.

Although poor quality of care decreases utilization of services [41], we found that most women in our study attended antenatal clinics at least four times during their pregnancy despite low provider adherence to guidelines during their first visit. It is uncertain how much of guideline requirement is known by clients, but one study has described high satisfaction for antenatal care services amongst attendants in Ghana [49]. Frequent antenatal attendance provides enough contact between the provider and the pregnant woman to identify and address any challenges of the pregnancy [50]. It is also important that during each of these contacts quality of care is assured.

We also find that women are reporting late for their first antenatal visit. Most women reported during the second trimester, as previously noted in studies both in Ghana and other low and middle-income countries [6, 51-55]. This is a source of concern as pregnancy complications may be recognized too late and the opportunity for timely interventions missed.

About $70 \%$ of the study participants had at least one form of complication during the antenatal, delivery or postpartum period. The majority of these complications occurred during antenatal period and anemia in pregnancy was high on the list. This is worrying in a setting where hemorrhage is a common cause of maternal deaths [56], since anemia will affect the woman's ability to adequately compensate physiologically in case of bleeding during delivery. We are not sure what proportion of this risk of anemia is due to physiological hemodilution in pregnancy [57], but malaria is a common cause of anemia in pregnancy in our setting [57-59], the incidence being $12.2 \%$ amongst our study participants. Both malaria in pregnancy and anemia potentially increase the risk of low birth weight and prematurity [58]. Compared to other studies, risk of hemorrhage was low (2.1\%) amongst our study participants compared to what other studies have found $[56,60]$. The relatively high rate of caesarean section in our study may partly be due to the fact many of our facilities were hospitals which receive referrals from lower level facilities. However, this was within the range of globally the acceptable rates of up to $15 \%[29,61]$.

Previous pregnancy history was found to be an important determinant of maternal outcome as shown in our study and other studies [62,63] and so providers can use this information in their clinical decision making during health care provision to pregnant women.

In the year 2013, the Greater Accra region recorded as many as 201 institutional maternal deaths [64]. For the 6 months of data collection within 11 facilities in the region no maternal death was recorded amongst our participants post-delivery. Maternal deaths are usually a result of direct causes like hemorrhage, pre-eclampsia and eclampsia and sepsis as well as indirect causes like anemia [65]. These occur mostly during delivery or within $24 \mathrm{~h}$ afterwards $[65,66]$. Although no maternal deaths occurred amongst our participants during the 6 weeks postpartum, it is possible that we missed some deaths that may have occurred before or during delivery since we recruited women who had already survived delivery or that our sample was not large enough to detect such an outcome in the postpartum period.

\section{Conclusion}

Complete provider adherence to first antenatal care guidelines was shown to influence neonatal outcomes but showed no effect on maternal outcomes. There is the need to explore and understand the possibility of explanatory mechanisms for these observations. Also, since adherence to first antenatal visit guidelines were used as a proxy for provider adherence to guidelines in general, we believe programs that promote complete adherence to guidelines will improve the outcome of neonatal service delivery. Early antenatal care should also be encouraged amongst the population.

\section{Additional file}

Additional file 1: Table showing the checklist for scoring provider adherence to antenatal guidelines. (DOCX $20 \mathrm{~kb}$ )

\section{Abbreviations}

ANC: Antenatal clinic; CHPS: Community based health and planning services; Cl: Confidence interval; ERC: Ethics review committee; GAR: Greater Accra Region; GHS: Ghana health service; IPTp: Intermittent presumptive treatment in pregnancy; MDG: Millennium development goal; NHIS: National Health Insurance Scheme; SD: Standard deviation; SMI: Safe motherhood initiative 


\section{Acknowledgement}

The authors gratefully acknowledge technical support from the Julius Center for Health Sciences and Primary Care, including Peter N. Zuithoff who provided support for statistical analysis. We also are grateful to the Ghana Health Service for the support given us to carry this research in its facilities. We also thank the Netherlands Organization for Scientific Research (NWO) Global Health Policy and 396 Health Systems Research Program, Netherlands, for providing funds for the conduct of the study (Grant number: 07.45.102.00).

\section{Funding}

Funding for this work was provided by the Netherlands Organization for Scientific Research (NWO) Global Health Policy and 396 Health Systems Research Program, Netherlands, for providing funds for the conduct of the study (Grant number: 07.45.102.00).

\section{Availability of data and materials}

This paper is part of a larger study for which ethical clearance from the Ghana Health Service Ethical Review Committee to collect and use the data for specific purposes was obtained. The data set is currently kept in our institutional database. The data will be available to any reader or researcher who meets the criteria for access to confidential data, upon approval by the Ghana Health Service Ethical Review Committee. All requests for the use of the data shall be made to the corresponding author of this paper (MAC) or the co-principal investigator of the larger study and co-author of this paper (EKA).

\section{Authors' contributions}

MAC designed and wrote up the study protocol, prepared it for ethical clearance, supervised data collection, carried out data analysis, wrote the report and drafted this manuscript for publication. EKA and KKG reviewed the protocol in preparation for ethical clearance, supervised data collection and manuscript preparation. GAK, IAA, DEG, EKA and KKG provided scientific guidance and review of the study design, data analysis and were also actively involved in the preparation and review of the manuscript and approved it. All authors read and approved the final manuscript.

\section{Competing interests}

The authors declare that they have no competing interests.

\section{Consent for publication}

Not applicable.

\section{Ethics approval and consent to participate}

The protocol, including a written consent document, was approved by the Ghana Health Service (GHS) Ethical Review Committee (ERC) under study ID No. GHS-ERC 12/07/2013 before the study was conducted. Also, the regional health directorate and heads of the health facilities gave permission for the conduct of the study. Written informed consent was obtained from each participant, including consent for data to be published.

\section{Author details}

'Postdoctoral Unit, Noguchi Memorial Institute for Medical Research, University of Ghana, Legon, Accra, Ghana. ${ }^{2}$ Julius Global Health, Julius Center for Health Sciences and Primary Care, University Medical Centre Utrecht, Utrecht, The Netherlands. ${ }^{3}$ Department of Epidemiology and Disease Control, School of Public Health, University of Ghana, Legon, Ghana. ${ }^{4}$ Division of Epidemiology \& Biostatistics, School of Public Health, Faculty of Health Sciences, University of the Witwatersrand, Johannesburg, South Africa. ${ }^{5}$ Department of Health Policy, Planning and Management, School of Public Health, University of Ghana, Legon, Ghana. ${ }^{6}$ Research and Development Division, Ghana Health Service, Accra, Ghana.

Received: 4 August 2015 Accepted: 16 November 2016 Published online: 24 November 2016

\section{References}

1. The World Bank - IBRD-IDA. Millennium Development Goals (Goal 5). 2016. Ref Type: Online Source. Accessed 6 Nov 2016

2. Campbell OM, Graham WJ. Strategies for reducing maternal mortality: getting on with what works. Lancet. 2006;368:1284-99.
3. Ministry of Health (MoH) GoGaUNCTitRoG. Ghana MDG acceleration fremawork and country action plan, maternal health. 2011. Ref Type: Repor. Accessed 8 Nov 2011.

4. Edmond KM, Zandoh C, Quigley MA, Amenga-Etego S, Owusu-Agyei S, Kirkwood BR. Delayed Breastfeeding Initiation Increases Risk of Neonatal Mortality. Pediatrics. 2006;117:e380-6.

5. Biritwum RB. Promoting and Monitoring Safe Motherhood in Ghana. Ghana Med J. 2006;40:78-9.

6. Pell C, Menaca A, Were F, Afrah NA, Chatio S, Manda-Taylor L, et al. Factors affecting antenatal care attendance: results from qualitative studies in Ghana, Kenya and Malawi. PLoS One. 2013;8:e53747.

7. WHO. Opportunities for Africa's newborns: Antenatal Care. 2013. Ref Type: Online Source.

8. Komajda M, Lapuerta P, Hermans N, Gonzalez-Juanatey JR, van Veldhuisen DJ, Erdmann E, et al. Adherence to guidelines is a predictor of outcome in chronic heart failure: the MAHLER survey. Eur Heart J. 2005;26:1653-9.

9. Cabana MD, Rand CS, Powe NR, Wu AW, Wilson MH, Abboud PA, et al. Why don't physicians follow clinical practice guidelines? A framework for improvement. JAMA. 1999:282:1458-65.

10. Folbert EC, Smit RS, van der Velde D, Regtuijt EM, Klaren MH, Hegeman JH. Geriatric fracture center: a multidisciplinary treatment approach for older patients with a hip fracture improved quality of clinical care and short-term treatment outcomes. Geriatr Orthop Surg Rehabil. 2012;3:59-67.

11. Wells K, Sherbourne C, Schoenbaum M, Ettner S, Duan N, Miranda J, et al. Five-year impact of quality improvement for depression: results of a group-level randomized controlled trial. Arch Gen Psychiatry. 2004;61:378-86.

12. Wells KB, Sherbourne C, Schoenbaum M, Duan N, Meredith L, Unutzer J, et al. Impact of disseminating quality improvement programs for depression in managed primary care: a randomized controlled trial. JAMA. 2000;283:212-20.

13. Abrams JA, Kapel RC, Lindberg GM, Saboorian MH, Genta RM, Neugut Al, et al. Adherence to biopsy guidelines for Barrett's esophagus surveillance in the community setting in the United States. Clin Gastroenterol Hepatol. 2009;7:736-42.

14. Adams AS, Soumerai SB, Lomas J, Ross-Degnan D. Evidence of self-report bias in assessing adherence to guidelines. Int J Qual Health Care. 1999:11:187-92.

15. Laye MR, Dellinger EH. Timing of scheduled cesarean delivery in patients on a teaching versus private service: adherence to American College of Obstetricians and Gynecologists guidelines and neonatal outcomes. Am J Obstet Gynecol. 2006;195:577-82.

16. Thudi K, Yadav D, Sweeney K, Behari J. Physicians infrequently adhere to hepatitis vaccination guidelines for chronic liver disease. PLoS One. 2013:8:e71124.

17. Fritz JM, Cleland JA, Brennan GP. Does adherence to the guideline recommendation for active treatments improve the quality of care for patients with acute low back pain delivered by physical therapists? Med Care. 2007:45:973-80.

18. Giorda C, Picariello R, Nada E, Tartaglino B, Marafetti L, Costa G, et al. The impact of adherence to screening guidelines and of diabetes clinics referral on morbidity and mortality in diabetes. PLoS One. 2012;7:e33839.

19. Johnstone J, Mandell L. Guidelines and quality measures: do they improve outcomes of patients with community-acquired pneumonia? Infect Dis Clin North Am. 2013;27:71-86.

20. Rice TW, Morris S, Tortella BJ, Wheeler AP, Christensen MC. Deviations from evidence-based clinical management guidelines increase mortality in critically injured trauma patients*. Crit Care Med. 2012;40:778-86.

21. Sales AE, Tipton EF, Levine DA, Houston TK, Kim Y, Allison J, et al. Are co-morbidities associated with guideline adherence? The MI-Plus study of Medicare patients. J Gen Intern Med. 2009;24:1205-10.

22. Lugtenberg M, Burgers JS, Westert GP. Effects of evidence-based clinical practice guidelines on quality of care: a systematic review. Qual Saf Health Care. 2009;18:385-92.

23. Okelo SO, Butz AM, Sharma R, Diette GB, Pitts SI, King TM, et al. Interventions To Modify Health Care Provider Adherence to Asthma Guidelines. Comp Eff Rev. 2013:132:517-34.

24. Vause J. Adherence to evidence-based guidelines is the key to improved health outcomes for general practice patients: YES. J Prim Health Care. 2012;4:156-8.

25. Sukums F, Mensah N, Mpembeni R, Massawe S, Duysburgh E, Williams A, et al. Promising adoption of an electronic clinical decision support system for antenatal and intrapartum care in rural primary healthcare facilities in sub-Saharan Africa: The QUALMAT experience. Int J Med Inform. 2015;84(9):647-57. 
26. Velding K, Klis SA, Abass KM, Tuah W, Stienstra Y, van der Werf T. Wound Care in Buruli Ulcer Disease in Ghana and Benin. Am J Trop Med Hyg. 2014;91:313-8

27. Chinbuah MA, Abbey M, Kager PA, Gyapong M, Nonvignon J, Ashitey P, et al. Assessment of the adherence of community health workers to dosing and referral guidelines for the management of fever in children under 5 years: a study in Dangme West District, Ghana. Int Health. 2013:5:148-56.

28. McKinlay JB, Link CL, Freund KM, Marceau LD, O'Donnell AB, Lutfey KL. Sources of variation in physician adherence with clinical guidelines: results from a factorial experiment. J Gen Intern Med. 2007;22:289-96.

29. Ghana Statistical Service. Ghana Demographic and Health Survey Report, 2008. Ref Type: Report. http://www.dhsprogram.com/pubs/pdf/FR221/ FR221[13Aug2012].pdf. Accessed 13 May 2012

30. WHO, UNFPA, UNICEF, World Bank. Pregnancy, Childbirth, Postpartum and Newborn Care: A guide for Essential Practice. Geneva: World Health Organization; 2006

31. Cuspidi C, Michev I, Lonati L, Vaccarella A, Cristofari M, Garavelli G, et al. Compliance to hypertension guidelines in clinical practice: a multicentre pilot study in Italy. J Hum Hypertens. 2002;16:699-703.

32. Advani A, Shahar Y, Musen MA: Medical quality assessment by scoring adherence to guideline intentions. Proc AMIA Symp 2001, 2-6

33. Van FE, Van Der Wee NJ, Bauer M, Witte CJ, Zitman FG. Assessing adherence to guidelines for common mental disorders in routine clinical practice. Int J Qual Health Care. 2012;24:72-9.

34. Amoakoh-Coleman M, Agyepong IA, Zuithoff NPA, Kayode GA, Grobbee DE, Klipstein-Grobusch K, et al. Client Factors Affect Provider Adherence to Clinical Guidelines during First Antenatal Care. PLoS One. 2016;11:e0157542.

35. Neupane S, Nwaru B. Impact of Prenatal Care Utilization on Infant Care Practices in Nepal: a National Representative Cross-sectional Survey. Eur J Pediatr. 2014;173:99-109.

36. Burstin HR, Conn AF, Setnik GF, Rucker DW FAU, Cleary PD FAU, O'Neil AC, FAU, et al. Benchmarking and quality improvement: the Harvard Emergency Department Quality Study. Am J Med. 1999;107(5):437-49.

37. Grilli R, Lomas J. Evaluating the Message: The Relationship between Compliance Rate and the Subject of a Practice Guideline. Med Care. 1994:32:202-13.

38. Scribano PV, Lerer T, Kennedy D, Cloutier MM. Provider Adherence to a Clinical Practice Guideline for Acute Asthma in a Pediatric Emergency Department. Acad Emergency Med. 2001;8(12):1147-52. doi:10.1111/j.15532712.2001.tb01131.x

39. Baker U, Tomson G, Som M, Kouyat B, Williams J, Mpembeni R, et al. 'How to know what you need to do': a cross-country comparison of maternal health guidelines in Burkina Faso, Ghana and Tanzania. Implement Sci. 2012;7:31.

40. Afulani PA. Rural/Urban and Socioeconomic Differentials in Quality of Antenatal Care in Ghana. PLoS One. 2015;10:e0117996.

41. Duysburgh E, Williams A, Williams J, Loukanova S, Temmerman M. Quality of antenatal and childbirth care in northern Ghana. BJOG. 2014;121:117-26.

42. Lomas J, Anderson GM, Domnick-Pierre K, Vayda E, Enkin MW, Hannah WJ. Do Practice Guidelines Guide Practice? N Engl J Med. 1989;321:1306-11.

43. Steinman MA, Fischer MA, Shlipak MG, Bosworth HB, Oddone EZ, Hoffman $\mathrm{BB}$, et al. Clinician awareness of adherence to hypertension guidelines. Am J Med. 2004;117:747-54.

44. Cheung A, Weir M, Mayhew A, Kozloff N, Brown K, Grimshaw J. Overview of systematic reviews of the effectiveness of reminders in improving healthcare professional behavior. Syst Rev. 2012;1:36.

45. Goldstein MK, Lavori PF, Coleman RF, Advani AF, Hoffman BB. Improving adherence to guidelines for hypertension drug prescribing: clusterrandomized controlled trial of general versus patient-specific recommendations. Am J Manag Care. 2005;11(11):677-85.

46. Grimshaw JM, Eccles MP, Walker AE, Thomas RE. Changing physicians' behavior: What works and thoughts on getting more things to work. J Contin Educ Health Prof. 2002;22:237-43.

47. Young $P$, Hamilton R, Hodgett S, Moss M, Rigby C, Jones $P$, et al. Reducing risk by improving standards of intrapartum fetal care. J R Soc Med. 2001;94:226-31.

48. Kardong-Edgren S. Using Evidence-Based Practice to Improve Intrapartum Care. J Obstet Gynecol Neonatal Nurs. 2001;30:371-5.

49. Degley J. Assessing The Quality Of Antenatal Care Services In The Nkwanta South District, Volta Region, Ghana. Accra: University of Ghana, Legon, Ghana; 2012.
50. WHO. Standards for Maternal and Neonatal care: Provision of effective antenatal care. 2013. p. 7-7-2014. Ref Type: Online Source.

51. Belayneh T, Adefris M, Andargie G. Previous Early Antenatal Service Utilization Improves Timely Booking: Cross-Sectional Study at University of Gondar Hospital, Northwest Ethiopia. J Pregnancy. 2014;2014:132494.

52. Doku D, Neupane S, Doku PN. Factors associated with reproductive health care utilization among Ghanaian women. BMC Int Health Hum Rights. 2012;12:29.

53. Haddrill R, Jones GL, Mitchell CA, Anumba DO. Understanding delayed access to antenatal care: a qualitative interview study. BMC Pregnancy Childbirth. 2014;14:207.

54. Ifenne DI, Utoo BT. Gestational age at booking for antenatal care in a tertiary health facility in north-central, Nigeria. Niger Med J. 2012;53:236-9.

55. Measure DHS. Ghana Demographic and Health Survey 2008. http://www. measuredhs.com/pubs/pdf/FR221.pdf.2011. 11-12-2011. Ref Type: Online Source

56. Asamoah BO, Moussa KM, Stafström M, Musinguzi G. Distribution of causes of maternal mortality among different socio-demographic groups in Ghana; a descriptive study. BMC Public Health. 2011;11:159.

57. Carroli G, Rooney C, Villar J. How effective is antenatal care in preventing maternal mortality and serious morbidity? An overview of the evidence. Paediatr Perinat Epidemiol. 2001;15:1-42.

58 Pell C, Meñaca A, Chatio S, Hodgson A, Tagbor H, Pool R. The acceptability of intermittent screening and treatment versus intermittent preventive treatment during pregnancy: results from a qualitative study in Northern Ghana. Malar J. 2014:13:432.

59 Stephens JK, Ofori MF, Quakyi IA, Wilson ML, Akanmori BD. Prevalence of peripheral blood parasitaemia, anaemia and low birthweight among pregnant women in a suburban area in coastal Ghana. Pan Afr Med J. 2014;17:3.

60 Ghana Health Service, USAID. Ghana Maternal Health Survey 2007 Report. Ref Type: Report. http://pdf.usaid.gov/pdf_docs/Pnado492.pdf. Accessed 3 May 2012.

61 WHO: Luz Gibbons JMBJALAPBMMaFA. The global numbers and costs of additionally needed and unnecessary caesarean sections performed per year: overuse as a barrier to universal coverage: World Health Report; 2010. Ref Type: Report. Accessed 23 Oct 2013

62 Jasovic-Siveska E, Jasovic VF, Stoilova S. Previous pregnancy history, parity, maternal age and risk of pregnancy induced hypertension.

63 Whitley E, Doyle P, Roman E, De Stavola B. The effect of reproductive history on future pregnancy outcomes. Hum Reprod. 1999;14:2863-7.

64 Mamaye! (Derick Adogla). Ghana: 1,012 Institutional Maternal Deaths recorded in 2013. 2014. Ref Type: Online Source. http://www.mamaye.org/ en/blog/ghana-1012-institutional-maternal-deaths-recorded-2013. Accessed 9 July 2015.

65 Nour NM. An Introduction to Maternal Mortality. Rev Obstet Gynecol. 2008;1:77-81.

66 Institute for Health Metrics and Evaluation (IHME). Maternal Mortality Estimates and MDG 5 Attainment by Country 1990-2011. Seattle: Institue for Health Metrics and Evaluation (IHME); 2011. Ref Type: Report.

\section{Submit your next manuscript to BioMed Central and we will help you at every step:}

- We accept pre-submission inquiries

- Our selector tool helps you to find the most relevant journal

- We provide round the clock customer support

- Convenient online submission

- Thorough peer review

- Inclusion in PubMed and all major indexing services

- Maximum visibility for your research

Submit your manuscript at www.biomedcentral.com/submit 